\title{
Optimizing Basal Insulin Delivery for Patients Suffering from Type I Diabetes Mellitus Using Particle Swarm Optimization Algorithm
}

\section{Aakash Sathe and Tesfay Meressi}

Department of Mechanical Engineering, University of Massachusetts Dartmouth, 285 Old Westport Road, North Dartmouth, Massachusetts 02747 , USA

"Corresponding author: Tesfay Meressi, Associate Provost for Graduate Studies, University of Massachusetts Dartmouth, 285 Old Westport Road, North Dartmouth, Massachusetts 02747, USA, Tel: 508-999-8542; E-mail: tmeressi@umassd.edu

Received Date: November 10, 2018; Accepted Date: November 25, 2018; Published Date: December 03, 2018

Copyright: ( 2018 Meressi T, et al. This is an open-access article distributed under the terms of the Creative Commons Attribution License, which permits unrestricted use, distribution, and reproduction in any medium, provided the original author and source are credited.

\begin{abstract}
Numerous attempts have been made at the design of controllers that can maintain glucose concentration levels in the body of a diabetes patient within ideal limits. Because of physiological differences, a control strategy that works for one patient may not work for another. There is a need to learn the glucose profile of a patient and come up with an optimum insulin delivery protocol specific to the patient. In this paper, we attempt to learn the glucose profile of a subject and compare it with an ideal/desired glucose profile. Using Particle Swarm Optimization (PSO) algorithm, we optimize the insulin delivery so that the glucose profile of the model of a subject being tested follows the ideal profile. The performance of the PSO algorithm is evaluated by varying some physiological parameters. Results show that the PSO algorithm is effective in maintaining the glucose concentration levels within the ideal range while following a pre-specified ideal profile.
\end{abstract}

Keywords: Diabetes mellitus; Hypoglycemia; Hyperglycemia; Particle swarm optimization; Basal insulin delivery

\section{Introduction}

According to the American Diabetes Association, the number of people diagnosed with diabetes is estimated to be over 150 million worldwide. By 2025, this number is expected to go up to 300 million [1]. In the United State alone, over 1.25 million people suffer from type I diabetes (T1D). The World Health Organisation describes diabetes as a chronic disease which severely affects the insulin production (type I) or the effectiveness of insulin in the body (type II). This disease is characterised by high concentration of blood glucose in the body. The glucose concentration in the body increases after the ingestion of a meal. In the case of healthy subjects, the $\beta$-cells in the pancreas release insulin when the blood glucose concentration increases. Insulin allows the cells to absorb glucose, leading to a decrease in the glucose concentration. However, for subjects suffering from Type I diabetes, the body loses its ability to produce insulin. This chronic disease destroys the $\beta$-cells which are responsible for the production of insulin in the body [2]. The destruction of $\beta$-cells results in excessive glucose concentration. This can lead to long-term complications which may include nerve damage, bone and joint problems, kidney failure and blindness.

In order to prevent such complications, insulin therapies which include injections, oral intake, and subcutaneous delivery by an insulin pump have been employed. These insulin therapies aim to mimic the insulin secretion process of the $\beta$-cells when the blood glucose concentration increases [2].

Research has shown that an oscillatory delivery of insulin is effective in maintaining the blood glucose concentration within the desired range $[2,3]$. The amount of insulin to be delivered is decided by medical practitioners depending on the metabolism and the physiological parameters of a patient.
This is usually done by trial and error until an insulin delivery protocol that results in acceptable levels of glucose concentration is determined. If the body does not receive the right amount of insulin, it can lead to both long and short-term health complications. A carefully designed protocol which delivers the optimum amount of insulin depending on the physiological parameters of a patient can reduce or prevent such complications.

In this paper, we use Particle Swarm Optimization (PSO) algorithm to optimize the insulin delivery by comparing the glucose profile of a patient's response to insulin delivery schedule with a pre-defined ideal profile. In order to simulate the insulin-glucose dynamics, we use a Delay Differential Equation (DDE) model for patients suffering from Type I Diabetes. We further study the effect of variation of physiological parameters and evaluate the performance of the optimization algorithm as measured by its ability to adjust the insulin delivery and track the ideal/desired glucose profile in the face of parameter variations.

\section{Materials and Methods}

\section{Mathematical model}

There has been a lot of research on the development of models that can describe the insulin-glucose kinetics effectively [4,5]. The Bergman model [6] is one of the most commonly used models to describe the insulin-glucose kinetics [7]. This model, also known as the Minimum Model, has parameters which are relatively easy to identify and is comparatively simple. However, the authors in [1,8-11] have highlighted the drawbacks and the possible shortcomings of this model. According to Al-Fandi et al. [1,8], this model fails to represent the oscillatory nature of the insulin-glucose system. One of the major drawbacks of the Bergman model is that it considers the insulinglucose system to be composed of two separate parts with a two-step process for parameter fitting. However, the authors in [9-11] argue that the system is an integrated physiological dynamic system, which 
Page 2 of 7

should be considered as a whole, with a single-step process for parameter fitting. This limits the model's use for effective controller design. Until 2008, the U.S. Food and Drug Administration (FDA) was using clinical trials to test if a product is safe to be used by diabetic patients. According to the authors [12], these trials failed to provide a reason as to why a product being launched was ineffective or unsafe. The use of computer simulators for in-silico tests has a great potential to advance research and accelerate innovation. The use of such computer models was approved by the FDA in 2008 [4], which included the model developed by Cobelli et al. [5] as a substitute for animal trials $[6,8]$. However, this model has several non-linear and algebraic equations along with a number of parameters which need to be identified, making it too complicated for control design.

This paper uses a Delay Differential model developed by Wang et al. [2] to describe the insulin-glucose dynamics for patients suffering from Type I Diabetes. This model was solved using the DDE 23 solver in MATLAB. Under the influence of external insulin infusion, the insulin-glucose dynamics are described using the following equations:

$$
\begin{gathered}
G^{\prime}=G_{\text {in }}(t)-f_{2}(G(t))-f_{3}(G(t)) f_{4}\left(I\left(t-\tau_{3}\right)\right)+f_{5}\left(I\left(t-\tau_{2}\right)\right)(1) \\
I^{\prime}=I_{\text {in }}(t)-d_{i} I(t)
\end{gathered}
$$

where, the insulin and glucose concentrations at any time $t \geq 0$ are given by $I(t)$ and $G(t)$ respectively. $I_{i n}(t)$ is the exogenous insulin intake rate while $G_{\text {in }}(t)$ denotes the glucose intake rate. The other source through which glucose enters the system is the liver. The a-cells located in the Langerhan's islets release the hormone glucagon when the plasma glucose concentration drops [2]. This hormone exerts control over the liver which leads to the production of glucose. The glucose production controlled by the insulin concentration is given by $\mathrm{f}_{5}(\mathrm{I})$, where, $\tau_{2}>0$ represents the hepatic glucose production delay. The insulin-independent glucose utilization, i.e. the glucose utilised by the brain and nerve cells is denoted by $f_{2}(G)$ while the insulin-dependent utilization, which is due to muscle, fat and other tissues, is denoted by $f_{3}(G) f_{4}(I)$. The time delay for insulin-dependent glucose utilization is given by $\tau_{3}>0$. The model developed by Sturis et al. [3] consisted of the function $f_{1}(G)$ to denote the insulin secretion as a function of glucose. The authors $[2,13]$ explain that the term $\operatorname{af}_{1}(G)$ denotes the pancreatic insulin delivery in a normal patient. The term $\alpha$ denotes the degree to which a patient is affected by diabetes. A patient whose pancreas has completely lost the ability to produce insulin will have $\alpha=0$. Wang et al. modified their model by omitting the function $\mathrm{f}_{1}$ and made appropriate changes to account for the insufficient insulin production by the pancreas [2]. The insulin clearance rate $\left(\mathrm{d}_{\mathrm{i}}\right)[2,14,15]$ represents the rate at which insulin is removed from the body $[15,16]$, $\mathrm{d}_{\mathrm{i}}$ is a constant and positive $\left(\mathrm{d}_{\mathrm{i}}>0\right)$. The insulin clearance rate plays an important role in controlling the insulin action. The functions $f_{2}$ through $\mathrm{f}_{5}$ are defined by the following equations.

$$
\begin{aligned}
& \left.\mathrm{f}_{2}(\mathrm{G})=\mathrm{U}_{\mathrm{b}}\left(1-\exp \left(-\mathrm{G} / \mathrm{C}_{2} \mathrm{~V}_{\mathrm{g}}\right)\right)\right) \\
& \mathrm{f}_{3}(\mathrm{G})=\mathrm{G} /\left(\mathrm{C}_{3} \mathrm{~V}_{\mathrm{g}}\right) \\
& \mathrm{f}_{4}(\mathrm{I})=\mathrm{U}_{0}+\left(\mathrm{U}_{\mathrm{m}}-\mathrm{U}_{0}\right) /\left(1-\exp \left(-\beta \ln \left(\mathrm{I} / \mathrm{C}_{4}\left(1 / \mathrm{V}_{\mathrm{i}}+1 /\left(0.2 \mathrm{t}_{\mathrm{i}}\right)\right)\right)\right)\right) \\
& \mathrm{f}_{5}(\mathrm{I})=\mathrm{R}_{\mathrm{g}} /\left(1+\exp \left(\hat{\alpha}\left(I / \mathrm{V}_{p}-\mathrm{C}_{5}\right)\right)\right)
\end{aligned}
$$

\begin{tabular}{|l|l|l|l|l|l|}
\hline Parameters & Units & Values & Parameters & Units & Values \\
\hline $\mathrm{V}_{\mathrm{g}}$ & $\mathrm{L}$ & 10 & $\mathrm{U}_{0}$ & $\mathrm{mg} \mathrm{min}^{-1}$ & 40 \\
\hline $\mathrm{U}_{\mathrm{b}}$ & $\mathrm{mg} \mathrm{min}^{-1}$ & 72 & $\mathrm{U}_{\mathrm{m}}$ & $\mathrm{mg} \mathrm{min}^{-1}$ & 940 \\
\hline
\end{tabular}

\begin{tabular}{|l|l|l|l|l|l|}
\hline $\mathrm{C}_{2}$ & $\mathrm{mg} \mathrm{l}^{-1}$ & 144 & $\beta$ & & 1.77 \\
\hline $\mathrm{C}_{3}$ & $\mathrm{mg} \mathrm{l}^{-1}$ & 1000 & $\mathrm{C}_{4}$ & $\mathrm{mU} \mathrm{l}^{-1}$ & 80 \\
\hline $\mathrm{V}_{\mathrm{p}}$ & $\mathrm{L}$ & 3 & $\mathrm{R}_{\mathrm{g}}$ & $\mathrm{mg} \mathrm{min}^{-1}$ & 180 \\
\hline $\mathrm{V}_{\mathrm{i}}$ & $\mathrm{L}$ & 11 & $\hat{\alpha}$ & ${\mathrm{I} \mathrm{U}^{-1}}$ & 0.29 \\
\hline $\mathrm{t}_{\mathrm{i}}$ & Min & 100 & $\mathrm{C}_{5}$ & $\mathrm{mUl}^{-1}$ & 26 \\
\hline
\end{tabular}

Table 1: Values of parameters in the functions defined by eqn. (3) through eqn. (6).

Typical values of the various parameters used in eqns. (3). through (6) are given in Table $1[2,3,14,15,17]$.

In this paper, we use insulin lispro, an ultra-rapid-acting insulin, for the treatment of Type I diabetes. Insulin lispro has a shorter duration of action and begins to reduce the blood sugar faster than regular insulin. It starts acting about $5 \mathrm{~min}$ after injection and reaches its peak effect at $30-90 \mathrm{~min}$. It usually has a lasting effect for 3-5 hours. It is assumed that a patient ingests a meal every four hours. The whole duration of glucose intake is $45 \mathrm{~min}$ and the glucose intake reaches it maximum value after $15 \mathrm{~min}$. It is further assumed that $G_{\text {in }}(t)$ and $\mathrm{I}_{\text {in }}(\mathrm{t})$ are piecewise linear functions which are periodic over a period $\omega$ $(\omega=240 \mathrm{~min}=4$ hours) [2].

Typical glucose and insulin infusion rate profiles are defined by the following equations

$$
\begin{gathered}
G_{\text {in }}(t)=\left\{\begin{array}{c}
0.05+\frac{5}{15} t, 0 \leq t<15(\mathrm{~min}) \\
0.05+5 \frac{45-t}{45-15}, 15 \leq t<45(\mathrm{~min}) \\
0.045,45 \leq t<240(\mathrm{~min})
\end{array}\right. \\
I_{\text {in }}(t)=\left\{\begin{array}{c}
0.25,0 \leq t<5(\mathrm{~min}) \\
0.25+\left(1+\frac{t-30}{30-5}\right), 5 \leq t<30(\mathrm{~min}) \\
0.25+\left(1-\frac{t-30}{120-30}\right), 30 \leq t<120(\mathrm{~min}) \\
0.25,120 \leq t<240(\mathrm{~min})
\end{array}\right.
\end{gathered}
$$

The glucose and insulin intake rates are shown in Figures 1 and 2 respectively.

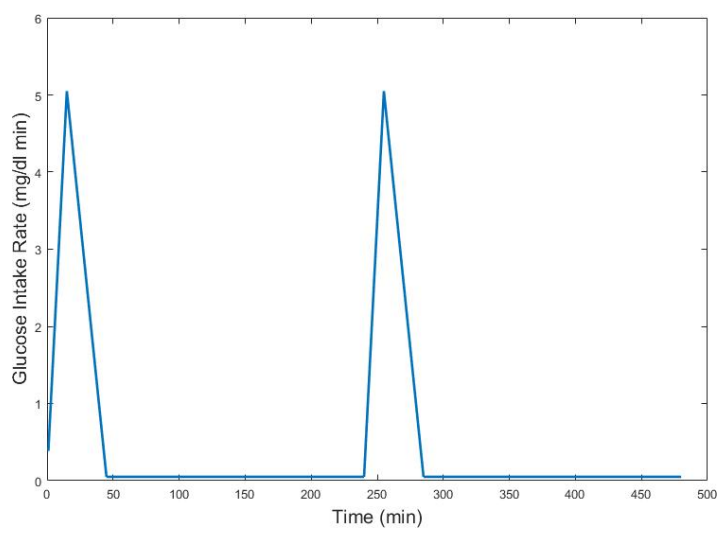

Figure 1: Glucose Intake Rate $G_{i n}$. 


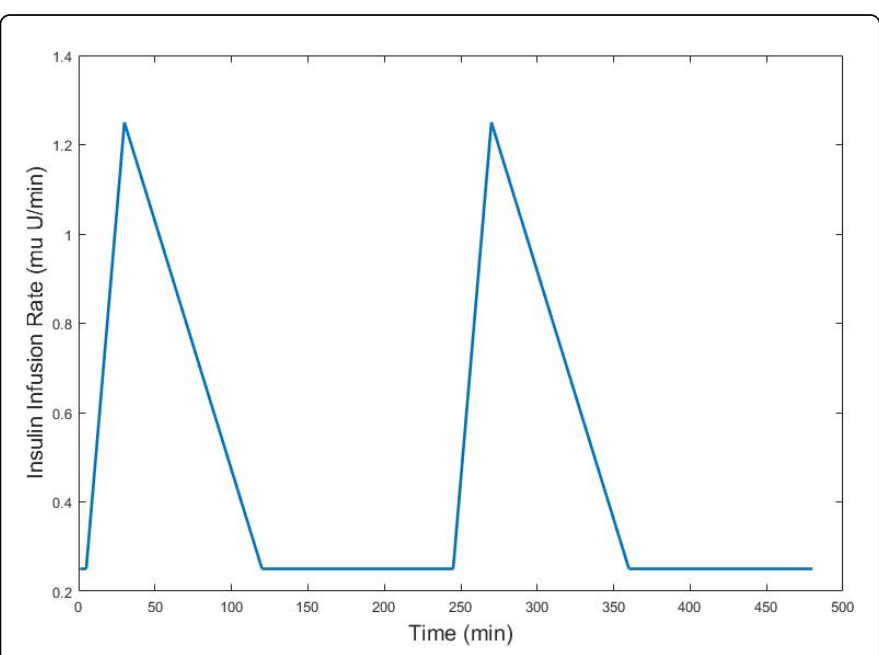

Figure 2: Insulin Infusion Rate $\mathrm{I}_{\mathrm{in}}$.

$\beta$-cells, found in the pancreas, are responsible for the production of insulin [2]. However, with the onset of Type I diabetes, these cells are completely destroyed and the insulin production is adversely affected. In the case of patients suffering from Type I Diabetes (T1D), the body loses its ability to produce insulin in 5-10 years [2]. This leads to excessive concentration of glucose in the bloodstream resulting in a harmful condition known as hyperglycemia. This is an undesirable condition which has long term effects on the body. To avoid this condition, it is important to administer insulin at regular time intervals. However, excessive amount of insulin can lead to a rapid decrease in the glucose concentration, leading to hypoglycemia, which is also harmful to the body. It is therefore very important to administer the right amount of insulin to maintain the glucose concentration within an ideal range.

In the following section, we introduce Particle Swarm Optimization (PSO) algorithm and its application in optimizing insulin delivery. The PSO algorithm is one of the most popular optimization algorithms because of its simplicity, speed of convergence and its ability to optimize different functions. We use this algorithm to study the glucose profiles of different patients, compare them to an ideal profile and optimize the exogenous insulin infusion rate $\left(\mathrm{I}_{\text {in }}\right)$ depending on the patient's physiological parameters.

\section{Particle swarm optimization}

Developed in 1995 by Kennedy and Eberhart [18-20], Particle Swarm Optimization (PSO) algorithm draws inspiration from swarm theory and the model of social interaction between different organisms. Its uniqueness is defined by its relation to genetic algorithms and evolutionary programming [19-22]. Unlike other optimization algorithms such as gradient descent and neural networks, particle swarm optimization eliminates the need to specify a gradient for the cost function. Because of its simplicity and its connection to genetic algorithms, it can be used to optimize different functions with ease [20]. Particle Swarm Optimization algorithm comprises of several vectors, known as "particles" which contain possible solutions to the optimization problem. These particles move in a multidimensional search space while retaining the memory of their best position and that of their neighbors. Every particle uses its memory to move towards the optimum solution and eventually converge at a certain point.
The velocity and position of every particle after each iteration is given by the following equations:

$$
\begin{aligned}
& \mathrm{v}_{\mathrm{ij}}(\mathrm{t}+1)=\mathrm{w}^{\star} \mathrm{v}_{\mathrm{ij}}(\mathrm{t})+\mathrm{r}_{1} \mathrm{c}_{1}\left(\mathrm{p}_{\mathrm{ij}}(\mathrm{t})-\mathrm{x}_{\mathrm{ij}}(\mathrm{t})\right)+\mathrm{r}_{2} \mathrm{c}_{2}\left(\mathrm{~g}_{\mathrm{i}}(\mathrm{t})-\mathrm{x}_{\mathrm{ij}}(\mathrm{t})\right) \\
& \mathrm{x}_{\mathrm{ij}}(\mathrm{t}+1)=\mathrm{x}_{\mathrm{ij}}(\mathrm{t})+\mathrm{v}_{\mathrm{ij}}(\mathrm{t}+1)
\end{aligned}
$$

where, $r_{1}$ and $r_{2}$ are two random numbers which are uniformly distributed in the range $(0,1)$ while $c_{1}$ and $c_{2}$ are the acceleration coefficients. The personal best position of each particle and the global best position of the swarm are given by "p" and "g" respectively. The parameter "w", also known as the inertia weight, was introduced in 1998 by Shi and Eberhart to improve the performance of the algorithm $[19,23]$. The value of the inertia weight defines the extent of exploration of the search space (Table 2).

\begin{tabular}{|l|l|}
\hline Swarm Size & 50 \\
\hline Number of Iterations & 100 \\
\hline Unknown Variables & 4 \\
\hline C1 & 2.05 \\
\hline C2 & 2.05 \\
\hline
\end{tabular}

Table 2: Parameters for PSO algorithm.

The equation to update the velocity as given by eqn. (9) consists of three main components: inertia component, cognitive component, and the social component. The first term in the equation represents the inertia component which prevents the particle from rapidly changing its direction. This term ensures that the particle retains the memory of its previous direction of travel. The second term, the cognitive component, enables the particle to acquire knowledge about its personal best positions and decide its direction in the search space. The third term represents the social component which ensures the collaboration of a particle with its neighbors.

In 2002, M. Clerc and J. Kennedy introduced the concept of constriction coefficients and their effect on the performance of PSO algorithm. They believed that the use of constriction coefficients would guarantee convergence and promote exploration of the search space $[22,23]$. The equation to update velocity as given in eqn. (9) was modified to include the constriction coefficient " $K$ " which is a function of the acceleration coefficients $c_{1}$ and $c_{2}$. The modified equation to update the velocity is given by:

$$
\begin{aligned}
& \mathrm{v}_{\mathrm{ij}}(\mathrm{t}+1)=\mathrm{K}^{\star}\left[\mathrm{v}_{\mathrm{ij}}(\mathrm{t})+\mathrm{r}_{1} \mathrm{c}_{1}\left(\mathrm{p}_{\mathrm{ij}}(\mathrm{t})-\mathrm{x}_{\mathrm{ij}}(\mathrm{t})\right)+\mathrm{r}_{2} \mathrm{c}_{2}\left(\mathrm{~g}_{\mathrm{i}}(\mathrm{t})-\mathrm{x}_{\mathrm{ij}}(\mathrm{t})\right)\right] \\
& K=\frac{2}{\left|2-\varnothing-\sqrt{\varnothing^{2}-4 \varnothing}\right|} \quad \varnothing=c_{1}+c_{2}>4
\end{aligned}
$$

\section{Validating the particle swarm optimization algorithm}

As mentioned before, Wang's model has been used as a reference model for our simulations. One typically used ideal glucose profile that limits glucose levels within the desired range is shown in Figure 3. However, since patients have different physiology, they require different insulin delivery profiles in order to keep their glucose levels within an ideal range. In this paper, we use Particle Swarm Optimization algorithm to determine the best insulin delivery for a patient so that the glucose profile follows a predefined ideal profile. 


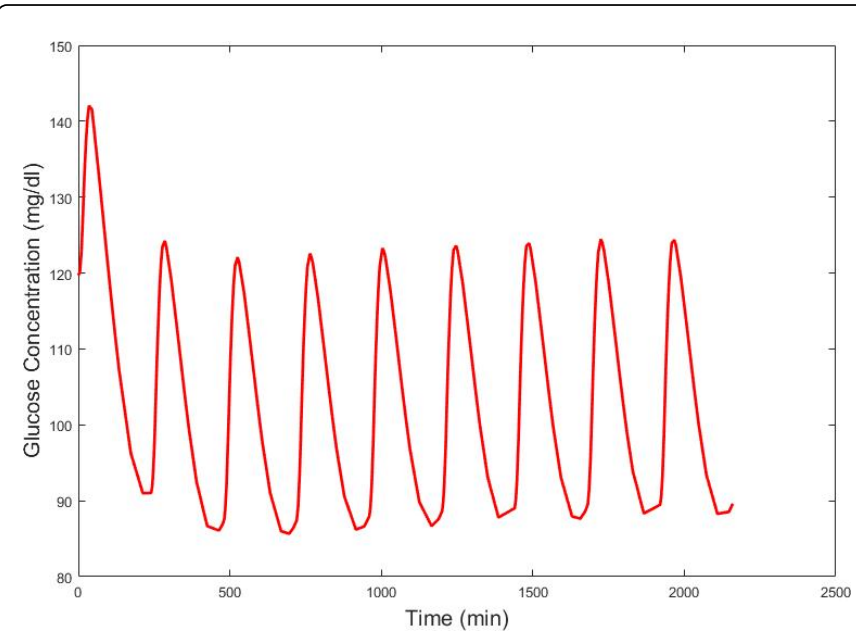

Figure 3: Ideal Glucose Profile.

A model having the same parameters as that of the reference model was used to validate the performance of the PSO algorithm. The PSO algorithm is used to minimize the overall sum of the instantaneous errors between the glucose profiles of the model under consideration and the reference model. The cost function (CF) is given by

$$
C F=\sum_{t=0}^{n}\left(\left(G_{\text {model }}-G_{\text {reference }}\right)^{2} / G_{\text {reference }}\right)
$$

where, $G_{\text {model }}$ is the glucose output of model of the subject being tested and $\mathrm{G}_{\text {reference }}$ is that of the ideal/reference model being used. The duration of simulation is given by $n$.

The goal of the PSO algorithm is to optimize the insulin delivery so that the model being tested has a glucose profile which is similar to that of the reference model. The PSO algorithm optimizes the parameters $\mathrm{a}, \mathrm{b}, \mathrm{c}$, and $\mathrm{d}$ of the insulin delivery profile given by:

$$
I_{\text {in }}(t)=\left\{\begin{array}{c}
a, 0 \leq t<b(\min ) \\
a+\left(1+\frac{t-c}{c-b}\right), b \leq t \leq c(\mathrm{~min}) \\
a+\left(1+\frac{t-c}{d-c}\right), c \leq t \leq d(\mathrm{~min}) \\
a, d \leq t \leq 240(\mathrm{~min})
\end{array}\right.
$$

As discussed in a study [21], actuators typically have saturation limits. To account for this, an insulin limiter is added to the actuator output. This limiter prevents the insulin infusion rate from rising above or falling below the specified limit.

$$
I_{\text {in }}(t)=\left\{\begin{array}{l}
1.50, I_{\text {in }} \geq 1.50 \\
0.25, I_{\text {in }} \leq 0.25
\end{array}\right.
$$

\section{Results and Discussion}

Figure 4 shows that the model used closely tracks the reference model which validates the performance of the PSO algorithm. The normalized error between the actual and the reference models after optimization was found to be $0.1318 \mathrm{mg} / \mathrm{dl}$. Figure 5 shows the reduction of the normalized error with every iteration. The insulin delivery profile parameters converged to the following values corresponding to the desired glucose profile.

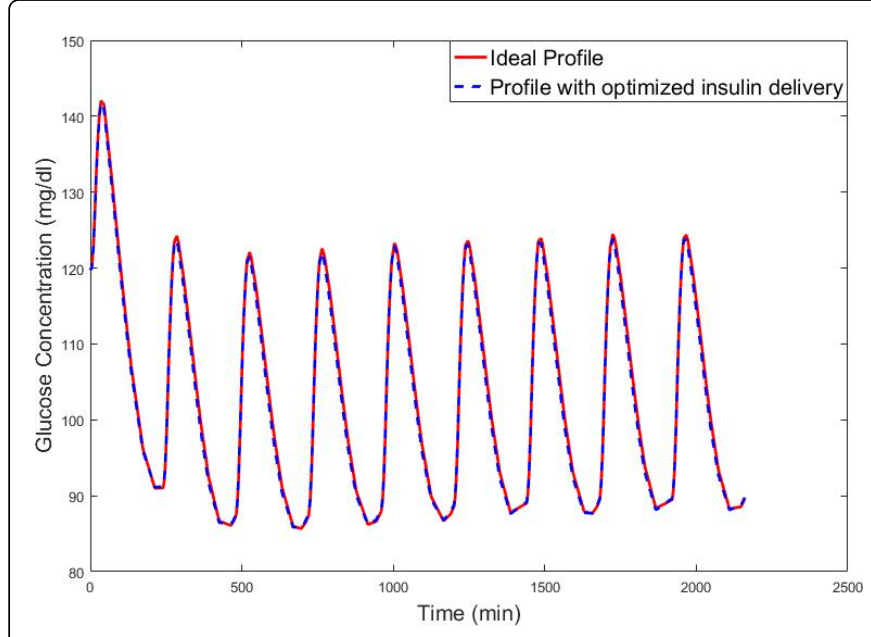

Figure 4: Glucose profiles of the PSO optimized system and the ideal model.

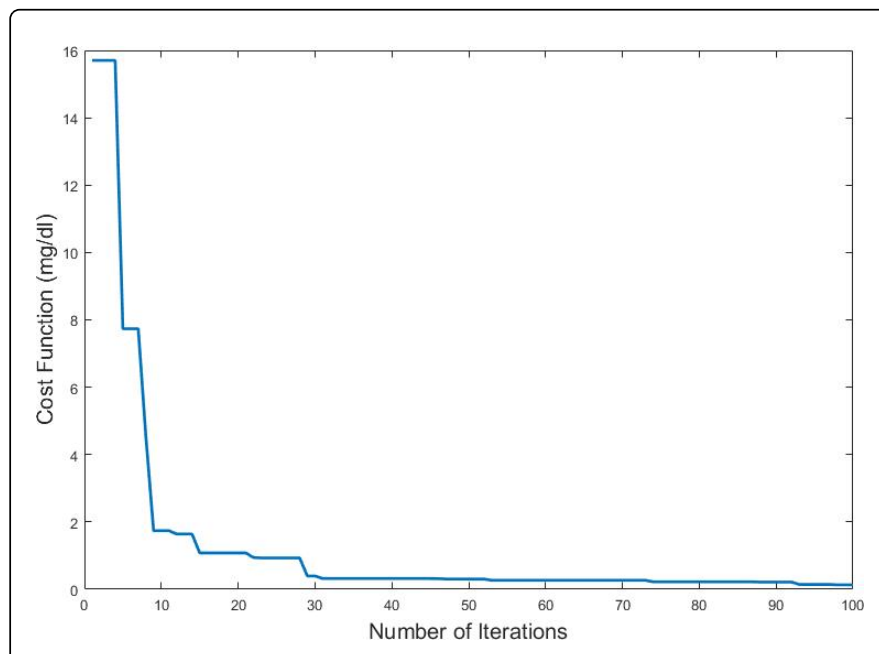

Figure 5: Reduction of total normalized error vs number of iterations.

$I_{\text {in }}(t)=\left\{\begin{array}{c}0.2606,0 \leq t \leq 8.33(\mathrm{~min}) \\ 0.2606+\left(1+\frac{t-30}{30-8.33}\right), 8.33 \leq t<30(\mathrm{~min}) \\ 0.2606+\left(1+\frac{t-30}{118.20-30}\right), 30 \leq t<118.20(\mathrm{~min}) \\ 0.2606,118.20 \leq t \leq 240(\mathrm{~min})\end{array}\right.$

Figure 6 shows the plot of the optimized insulin infusion rate based on eqns. (15) and (16). It can be seen that the use of the optimized insulin delivery parameters leads to an insulin delivery profile similar to the original insulin delivery profile shown in Figure 2. 
Page 5 of 7

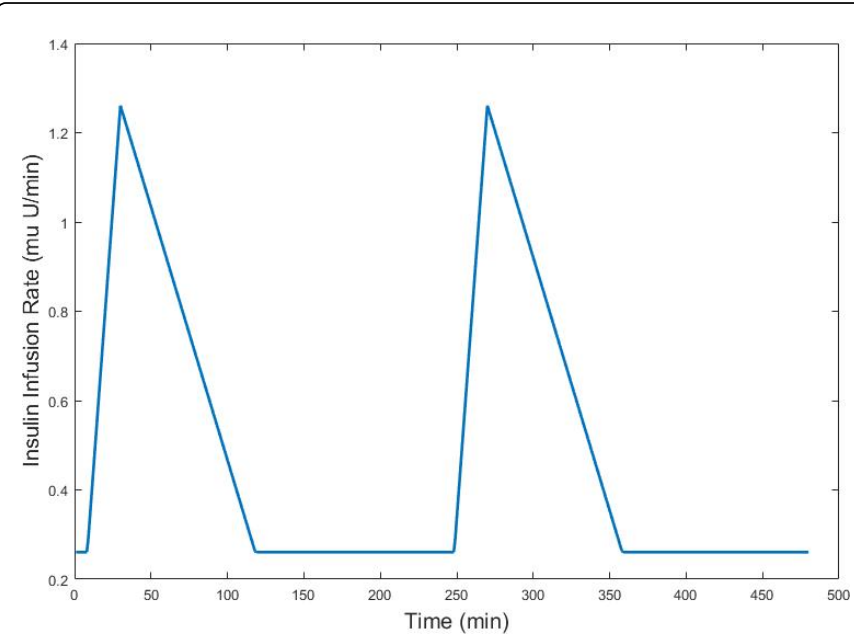

Figure 6: Optimized Insulin Infusion Rate.

In the following section, we describe the robustness of the algorithm to variations in the clearance rate $\left(\mathrm{d}_{\mathrm{i}}\right)$ and the rate at which the algorithm adjusts the insulin delivery profile.

\section{Robustness to variation in the clearance rate}

The value of the clearance rate $\left(\mathrm{d}_{\mathrm{i}}\right)$ varies from patient to patient and it is important to test the robustness of the algorithm against this variation. Changing the clearance rate directly affects the insulin absorption. To account for the variation in insulin absorption rate for different subjects, the clearance rate $\left(\mathrm{d}_{\mathrm{i}}\right)$ was varied from its nominal value by $20 \%$. The nominal value of $d_{i}$ was considered to be 0.0076 as stated in a study [2]. As was done in a study [8] we assumed the acceptable range of glucose concentration to be between $60-140 \mathrm{mg} / \mathrm{dl}$.

$\mathrm{d}_{\mathrm{i}}=0.0076 \pm 20 \%$

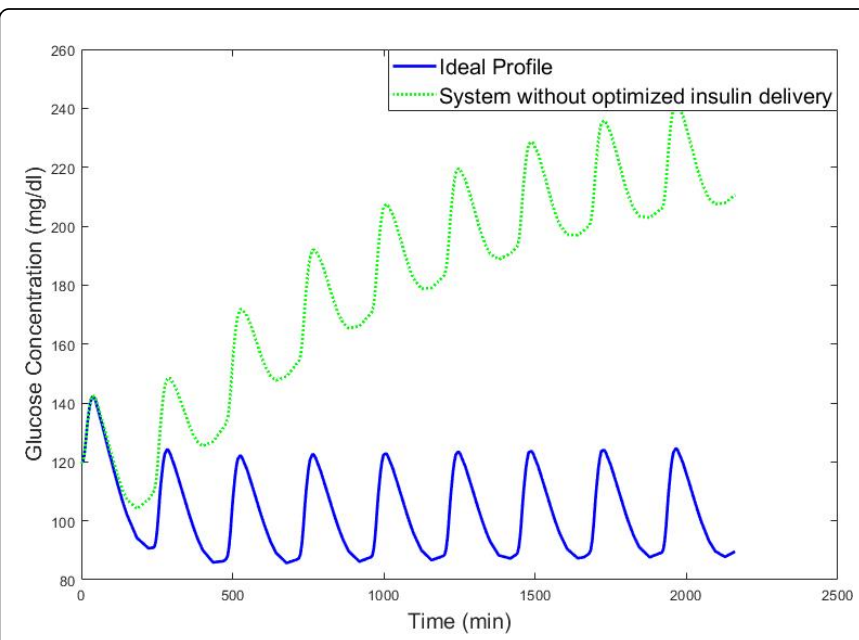

Figure 7: Glucose Profile of the reference model when $d_{i}$ is increased by $20 \%$.

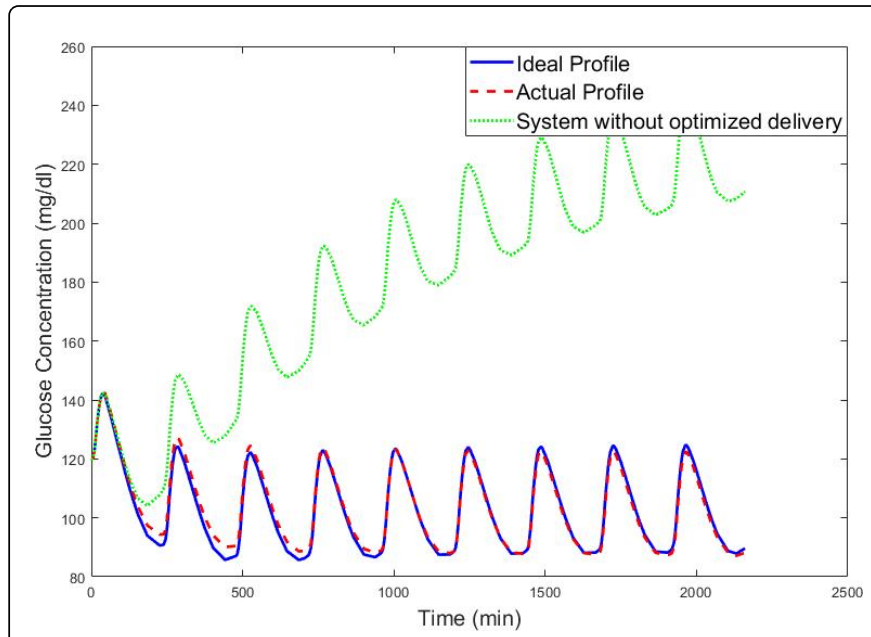

Figure 8: Comparison of glucose profiles of the reference model and the PSO optimized system when $\mathrm{d}_{\mathrm{i}}=0.00912$.

Figure 7 shows the glucose profile corresponding to the nominal model with a $20 \%$ increase in $d_{i}$ while Figure 8 compares the glucose profile of the reference model with those corresponding to PSOoptimized and non-optimized insulin delivery. When $\mathrm{d}_{\mathrm{i}}$ was increased by $20 \%$, insulin was cleared at a faster rate from the system. The reference model was being supplied a pre-determined amount of insulin as given by eqn. (8). This time-dependent insulin delivery failed to maintain acceptable glucose range in the face of the modelling error. This resulted in elevated glucose levels which led to hyperglycemia. Since the PSO algorithm aims at minimizing the error between the glucose concentration of the model being tested and the ideal glucose profile, it generates optimized values of the insulin delivery parameters in eqn. (14). Figure 8 shows how well the glucose profile of the optimized system was able to follow the ideal glucose profile while staying within acceptable range. The total error corresponding to the PSO optimized insulin delivery was $5.193 \mathrm{mg} / \mathrm{dl}$ as shown in Figure 9. The insulin delivery profile corresponding to the optimized parameters when $d_{i}$ was increased by $20 \%$ is given by:

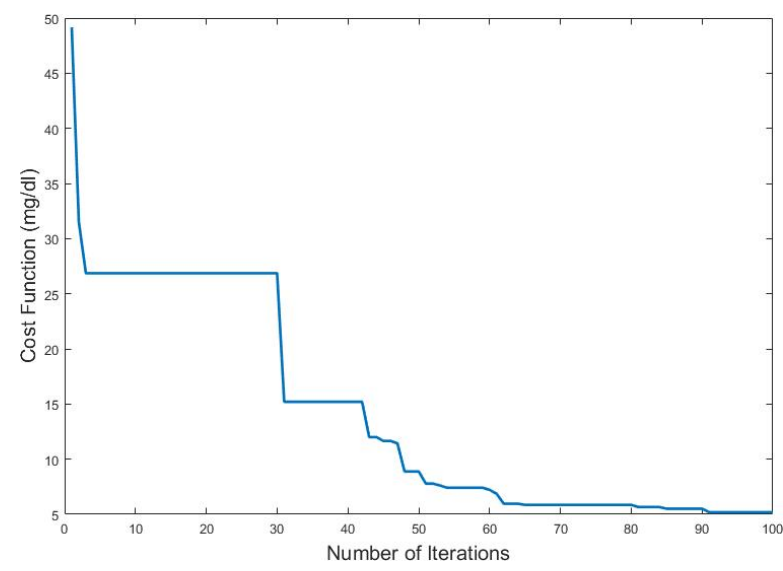

Figure 9: Reduction of total normalized error when $\mathrm{d}_{\mathrm{i}}=0.00912$. 


$$
I_{\text {in }}(t)=\left\{\begin{array}{c}
0.3841,0 \leq t \leq 14.18(\mathrm{~min}) \\
0.3841+\left(1+\frac{t-30}{30-14.18}\right), 14.18 \leq t<30(\mathrm{~min}) \\
0.3841+\left(1+\frac{t-30}{111.60-30}\right), 30 \leq t<111.60(\mathrm{~min}) \\
0.3841,111.60 \leq t \leq 240(\mathrm{~min})
\end{array}\right.
$$

When $d_{i}$ was decreased by $20 \%$, the rate of clearance of insulin from the system was very slow. Although insulin was cleared from the system at a slower rate, the body still received the same amount of insulin according to eqn. (8). This led to a condition known as hypoglycemia, which is characterised by low glucose concentration. As shown in Figure 10, the glucose concentration of the reference model fell below $60 \mathrm{mg} / \mathrm{dl}$ which is not desirable.

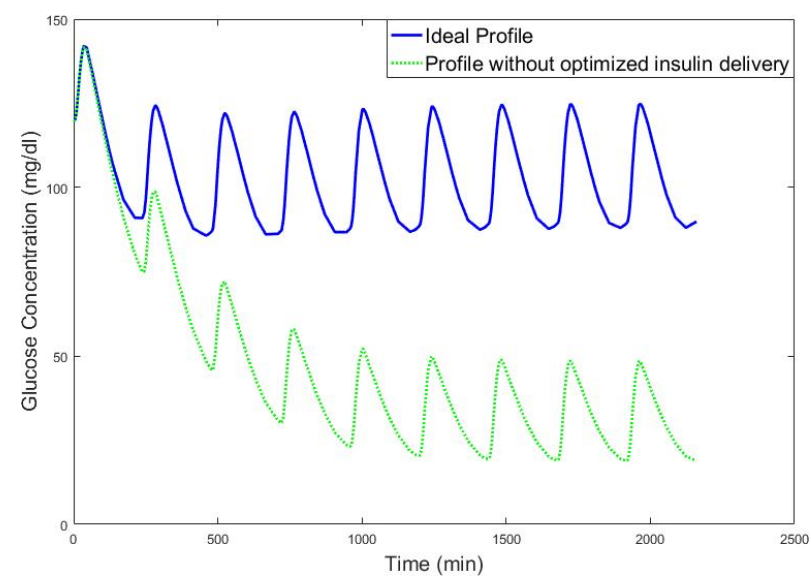

Figure 10: Glucose Profile of the reference model when di is decreased by $20 \%$.

The insulin delivery profile corresponding to the optimized parameters when $d_{i}$ was decreased by $20 \%$ is given by:

$I_{\text {in }}(t)=\left\{\begin{array}{c}0.25,0 \leq t \leq 12.69(\mathrm{~min}) \\ 0.25+\left(1+\frac{t-37.15}{37.15-12.69}\right), 12.69 \leq t<37.15(\mathrm{~min}) \\ 0.3841+\left(1+\frac{t-37.15}{76.21-37.15}\right), 37.15 \leq t<76.21(\mathrm{~min}) \\ 0.25,76.21 \leq t \leq 240(\mathrm{~min})\end{array}\right.$

The algorithm was able to optimize the insulin delivery so that the glucose profile of the model of the subject being tested could follow the ideal profile as shown in Figure 11. The total normalized error after optimization was $4.2807 \mathrm{mg} / \mathrm{dl}$ as shown in Figure 12. It can be seen that, while maintaining the glucose levels within the ideal range, the PSO algorithm was able to adapt to the variation in the clearance rate effectively.

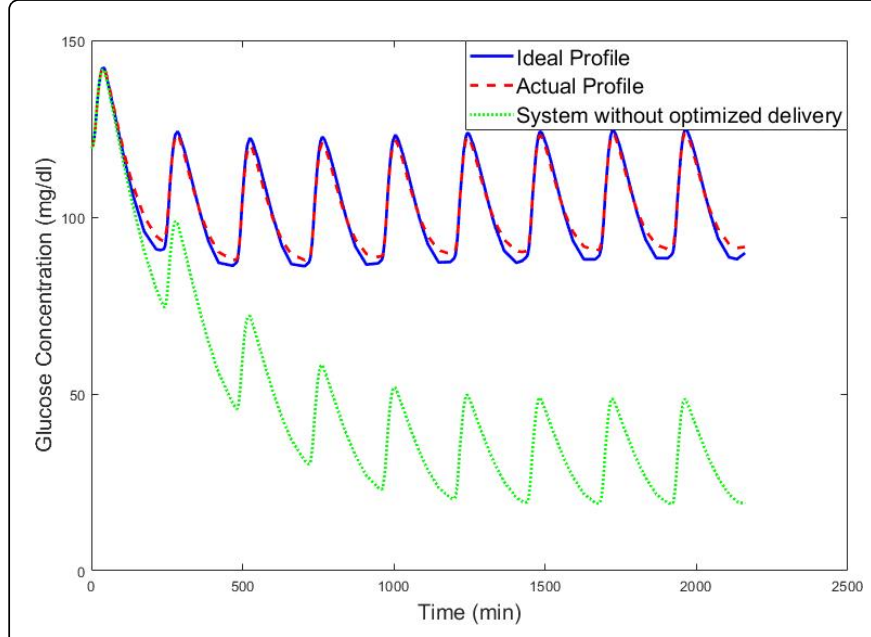

Figure 11: Comparison of glucose profiles of the reference model and the PSO optimized system when $\mathrm{d}_{\mathrm{i}}=0.00608$.

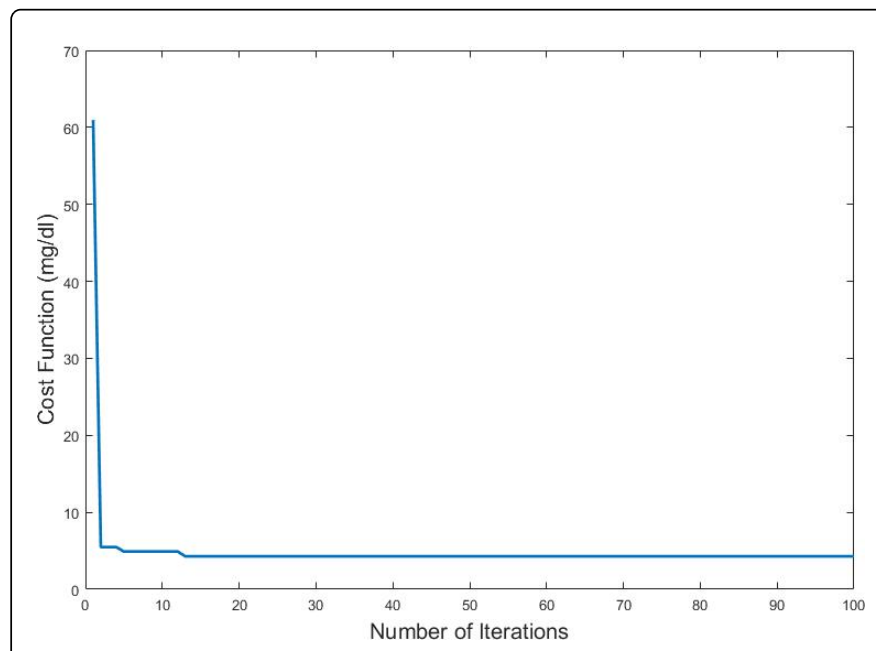

Figure 12: Reduction of total normalized error when $\mathrm{d}_{\mathrm{i}}=0.00608$.

\section{Conclusion}

A Particle Swarm Optimization (PSO) algorithm has been proposed to optimize insulin delivery to patients with type I diabetes. A delay differential model developed by Wang et al. is used to describe the dynamics of the insulin-glucose system. The goal of the algorithm is to mimic the ideal/desired glucose profile of a healthy subject. The algorithm aims to optimize insulin delivery by reducing the error between the glucose profile of the subject and the ideal/desired profile. To test the robustness of the algorithm to varying physiological parameters of patients, the clearance rate was varied by $20 \%$.

The results presented in this paper show that the algorithm is effective in dealing with parameter variations while maintaining the blood glucose levels within the desired range. The desired periodic insulin delivery profile mimics the oscillatory nature of the insulinglucose system corresponding to normal pancreatic insulin delivery function. The results show that the PSO algorithm provides the flexibility of adjusting the insulin infusion rate depending on the 
Citation: Sathe A, Meressi T (2018) Optimizing Basal Insulin Delivery for Patients Suffering from Type I Diabetes Mellitus Using Particle Swarm Optimization Algorithm. J Bioengineer \& Biomedical Sci 8: 256. doi:10.4172/2155-9538.1000256

Page 7 of 7

glucose profile response of the subject. This optimization tool can help medical practitioners decide the best patient-specific insulin delivery profile that can be used to deliver a continuous basal insulin dose throughout the day.

\section{References}

1. Al-Fandi M, Jaradat MA, Sardahi Y (2012) Optimal PID-Fuzzy logic controller for type 1 diabetic patients. proceedings of the international symposium on mechatronics and its applications, United Arab Emirates.

2. Wang H, Li J, Kuang Y (2007) Mathematical modeling and qualitative analysis of insulin therapies. Math Biosci 210: 17-33.

3. Sturis J, Polonsky KS, Mosekilde E, Van Cauter E (1991) Computer model for mechanisms underlying ultradian oscillations of insulin and glucose. Am J Physiol 260: E801-E809

4. Man CD, Rizza RA, Cobelli C (2007) Meal simulation model of the glucose insulin system. IEEE Trans Biomed Eng 54: 1740-1749.

5. Cobelli C, Man C, Sparacino G, Magni L, Nicolao G, et al. (2009) Diabetes: models, signals and controllers. IEEE Rev Biomed Eng 2: 54-96.

6. Bergman RN, Phillips LS, Cobelli C (1981) Physiologic evaluation of factors controlling glucose tolerance in man: measurement of insulin sensitivity and $\beta$-cell glucose sensitivity from the response to intravenous glucose. J Clin Invest 68: 1456-1467.

7. Li P, Yu L, Guo L, Dong J, Hu J, et al. (2012) PID control of glucose concentration in subjects with type 1 diabetes based on a simplified model: an in-silico trial. Intelligent Control and Automation (WCICA), 10th World Congress on, 2012. IEEE. China, pp: 5051-5055

8. Al-Fandi M, Jaradat M, Sardahi Y (2011) Optimal PI-fuzzy logic controller of glucose concentration using genetic algorithm. International Journal of Knowledge-Based and Intelligent Engineering Systems 15: 99-117.

9. Wilinska ME, Hovorka R (2008) Simulation models for in silico testing of closed-loop glucose controllers in type 1 diabetes. Drug Discovery Today: Disease Models 5(4); 289-298.

10. Li J, Kuang Y, Li B (2001) Analysis of IVGTT glucose-insulin interaction models with time delay. Discrete and Continuous Dynamical SystemsSeries B 1(1): pp.103-124.
11. De Gaetano A, Arino O (2000) Mathematical modeling of the intravenous glucose tolerance test. J Math Biol 40: 136-168.

12. Viceconti M, Henney A, Morley-Fletcher E (2016) In silico clinical trials: how computer simulation will transform the biomedical industry. Int J Clin Trials 3: 37-46

13. Engelborghs K, Lemaire V, Belair J, Roose D (2001) Numerical bifurcation analysis of delay differential equations arising from physiological modelling. J Math Biol 42: 361-385.

14. Li J, Kuang Y (2007) Analysis of a model of the glucose-insulin regulatory system with two delays. SIAM J Appl Math 67: 757-776.

15. Li J, Kuang Y, Mason C (2006) Modeling the glucose-insulin regulatory system and ultradian insulin secretory oscillations with two time delays. J Theor Biol 242: 722-735.

16. Duckworth W, Bennett R, Hamel F (1998) Insulin degradation: progress and potential. Endocr Rev 19: 608-624.

17. Tolic I, Mosekilde E, Sturis J (2000) Modeling the insulin-glucose feedback system: the significance of pulsatile insulin secretion. J Theor Biol 207: 361-375.

18. Kennedy J, Eberhart R (1995) Particle swarm optimization. Proceedings of the IEEE International Conference on Neural Networks, Australia.

19. Shi Y, Eberhart R (1998) A Modified Particle Swarm Optimizer. Proceedings of the 1998 IEEE International Conference on Evolutionary Computation, USA.

20. Eberhart R, Kennedy J (1995) A new optimizer using particle swarm theory. Proceedings of the sixth international symposium on micro machine and human science, Japan.

21. Khooban M, Abadi D, Alfi A, Siahi M (2013) Swarm optimization tuned Mamdani fuzzy controller for diabetes delayed model. Turkish Journal of Electrical Engineering and Computer Sciences, 21: 2110-2126.

22. Clerc M, Kennedy J (2002) The particle swarm-explosion, stability, and convergence in a multidimensional complex space. IEEE Transactions on Evolutionary Computation 6: 58-73.

23. Eberhart R, Shi Y (2000) Comparing inertia weights and constriction factors in particle swarm optimization. Proceedings of the 2000 Congress on Evolutionary Computation. 